\title{
Light hadron spectrum with 2+1 flavor dynamical $O(a)$-improved Wilson quarks
}

\author{
PACS-CS Collaboration: N. Ukita ${ }^{*} \dagger$ S. Aoki ${ }^{b, c}$, N. Ishii ${ }^{a}$, K.-I. Ishikawa ${ }^{d}$, \\ N. Ishizuka ${ }^{a, b}$, T. Izubuchi ${ }^{c, e}$, D. Kadoh ${ }^{a}$, K. Kanaya ${ }^{b}$, Y. Kuramashi ${ }^{a, b}$, Y. Namekawa ${ }^{a}$, \\ M. Okawa ${ }^{d}$, K. Sasaki. ${ }^{a}$, Y. Taniguchi ${ }^{a, b}$, A. Ukawe ${ }^{a, b}$, T. Yoshié Th $^{a, b}$ \\ ${ }^{a}$ Center for Computational Sciences, University of Tsukuba, Tsukuba, Ibaraki 305-8577, Japan \\ ${ }^{b}$ Graduate School of Pure and Applied Sciences, University of Tsukuba, Tsukuba, Ibaraki \\ 305-8571, Japan \\ ${ }^{c}$ Riken BNL Research Center, Brook-haven National Laboratory, Upton, New York 11973, USA \\ ${ }^{d}$ Department of Physics, Hiroshima University, Higashi-Hiroshima, Hiroshima 739-8526, Japan \\ ${ }^{e}$ Institute for Theoretical Physics, Kanazawa University, Kanazawa, Ishikawa 920-1192, Japan
}

\begin{abstract}
We present preliminary results for the light harden spectrum in $N_{f}=2+1$ lattice QCD using the nonperturbatively $O(a)$-improved Wilson quark action and the Iwasaki gauge action. Simulations are carried out at $\beta=1.90$ on a $32^{3} \times 64$ lattice using the PACS-CS computer. We employ Lüscher's domain-decomposed HMC algorithm to reduce the up-down quark masses toward the physical value. The pseudoscalar meson masses range from $730 \mathrm{MeV}$ down to $210 \mathrm{MeV}$. We compare the light harden spectrum extrapolated to the physical point with the experimental values.
\end{abstract}

The XXV International Symposium on Lattice Field Theory July 30-4 August 2007 Regensburg, Germany

\footnotetext{
* Speaker.

†E-mail: ukita@ccs.tsukuba.ac.jp
} 


\section{Introduction}

It is an essential step for lattice QCD calculation to reproduce the harden spectrum with various systematic errors under full control and show that QCD is the fundamental theory of the strong interaction. Recent developments in simulation algorithms and computational facilities now allow us to remove the most troublesome systematic errors: the quenching effect and chiral extrapolation. The main task of the previous CP-PACS/JLQCD project [1] 2] was to remove the former by performing $N_{f}=2+1$ lattice QCD simulations with the nonperturbatively $O(a)$-improved Wilson quark action [3] and the Iwasaki gauge action [4] on a $(2 \mathrm{fm})^{3}$ lattice at three lattice spacings. The lightest up-down quark mass, however, was still $64 \mathrm{MeV}$ corresponding to $m_{\pi} / m_{\rho} \approx 0.6$ so that there remained an ambiguity from a long chiral extrapolation.

In the PACS-CS(Parallel Array Computer System for Computational Science) project [5, 6, ,7, 8], we aim to perform $N_{f}=2+1$ lattice QCD simulations at the physical point using the PACS-CS computer with a total peak speed of 14.3 TFLOPS developed and installed at University of Tsukuba on 1 July 2006. The quark and gauge actions are the same as in the previous CP-PACS/JLQCD project. To reduce the degenerate up and down quark masses we employ the domain-decomposed HMC (DDHMC) algorithm with the replay trick proposed by Lüscher [9] 10], whose effectiveness for small quark mass region has already been shown in the $N_{f}=2$ case [9 11] incorporating the multiple time scale integration scheme [12]. The strange quark is included by the UV-filtered Polynomial Hybrid Monte Carlo (UV-PHMC) algorithm [13].

In this report we present simulation details and some preliminary results for the hadron spectrum. The analysis on the chiral behavior of the pseudoscalar meson masses and the decay constants using chiral perturbation theory is given in a separate report [14].

\section{Simulation details}

We employ the $O(a)$-improved Wilson quark action with a nonperturbative improvement coefficient $c_{\mathrm{SW}}=1.715$ [15] and the Iwasaki gauge action at $\beta=1.90$ on a $32^{3} \times 64$ lattice. Table 1 . summarizes our simulation parameters. Simulations are carried out for six combinations of the hopping parameters $\left(\kappa_{\mathrm{ud}}, \kappa_{\mathrm{s}}\right)$. The heaviest case of $\left(\kappa_{\mathrm{ud}}, \kappa_{\mathrm{s}}\right)=(0.13700,0.13640)$ is chosen for a direct comparison of the PACS-CS results with the previous CP-PACS/JLQCD ones which em-

\begin{tabular}{ccccccc}
\hline$\kappa_{\text {ud }}$ & $\kappa_{\mathrm{s}}$ & $\tau$ & $\left(N_{0}, N_{1}, N_{2}\right)$ & $N_{\text {poly }}$ & MD time & $\tau_{\text {int }}[P]$ \\
\hline \hline 0.13700 & 0.13640 & 0.5 & $(4,4,10)$ & 180 & 2000 & $38.2(17.3)$ \\
0.13727 & 0.13640 & 0.5 & $(4,4,14)$ & 180 & 2000 & $20.9(10.2)$ \\
0.13754 & 0.13640 & 0.5 & $(4,4,20)$ & 180 & 2500 & $19.2(8.6)$ \\
0.13770 & 0.13640 & 0.25 & $(4,4,16)$ & 180 & 2000 & $38.4(25.2)$ \\
0.13781 & 0.13640 & 0.25 & $(4,4,48)$ & 180 & 350 & $9.1(6.1)$ \\
\hline 0.13754 & 0.13660 & 0.5 & $(4,4,28)$ & 220 & 900 & $10.3(2.9)$ \\
\hline
\end{tabular}

Table 1: Summary of simulation parameters. MD time is the number of trajectories multiplied by the trajectory length $\tau . \tau_{\text {int }}[P]$ denotes the integrated autocorrelation time for the plaquette. 
ployed this parameter as the smallest quark mass. Among two choices of $\kappa_{\mathrm{s}}, \kappa_{\mathrm{s}}=0.13640$ is the physical point $\kappa_{\mathrm{s}}=0.136412(50)$ as determined in the previous CP-PACS/JLQCD work [1], while $\kappa_{\mathrm{s}}=0.13660$ is to investigate the strange quark mass dependence.

The DDHMC algorithm is implemented by domain-decomposing the full lattice with a $8^{4}$ block size as a preconditioner for HMC. The domain-decomposition factorizes the up-down quark determinant to the UV and the IR parts distinctively. On the other hand, the UV-PHMC algorithm for the strange quark is not domain-decomposed. We can incorporate the multiple time scale integration scheme in these algorithms to reduce the simulation cost efficiently. The relative magnitudes of the force terms are found to be

$$
\left\|F_{\mathrm{g}}\right\|:\left\|F_{\mathrm{UV}}\right\|:\left\|F_{\mathrm{IR}}\right\| \approx 16: 4: 1,
$$

where $F_{\mathrm{g}}$ denotes the gauge part and $F_{\mathrm{UV}, \mathrm{IR}}$ for the UV and the IR parts of the up-down quarks. We write the associated step sizes for the above forces as $\delta \tau_{\mathrm{g}}=\tau / N_{0} N_{1} N_{2}, \quad \delta \tau_{\mathrm{UV}}=\tau / N_{1} N_{2}, \delta \tau_{\mathrm{IR}}=$ $\tau / N_{2}$ with $\tau$ the trajectory length, where the integers $N_{0}, N_{1}, N_{2}$ are chosen such that

$$
\delta \tau_{\mathrm{g}}\left\|F_{\mathrm{g}}\right\| \approx \delta \tau_{\mathrm{UV}}\left\|F_{\mathrm{UV}}\right\| \approx \delta \tau_{\mathrm{IR}}\left\|F_{\mathrm{IR}}\right\|
$$

We take $N_{0}=N_{1}=4$. Since we have found $\left\|F_{\mathrm{S}}\right\| \approx\left\|F_{\mathrm{IR}}\right\|$ for the strange quark part, we choose $\delta \tau_{\mathrm{s}}=\delta \tau_{\mathrm{IR}}$. The value of $N_{2}$ and the polynomial order for UV-PHMC $N_{\text {poly }}$ are adjusted taking account of acceptance rate and simulation stability.

The inversion of the Wilson-Dirac operator $D$ is carried out by the GCR solver with the stopping condition $|D x-b| /|b|<10^{-9}$ for the force calculation and $10^{-14}$ for the Hamiltonian, which guarantees the reversibility of the molecular dynamics trajectories to high precision: $|\Delta U|<10^{-12}$ for the link variables and $|\Delta H|<10^{-8}$ for the Hamiltonian.

\section{Plaquette history and autocorrelation time}

In Fig. 1 1 we show the plaquette history and the normalized autocorrelation function at $\left(\kappa_{\mathrm{ud}}, \kappa_{\mathrm{s}}\right)=$ $(0.13727,0.13640)$ as a representative case. The integrated autocorrelation time is estimated as
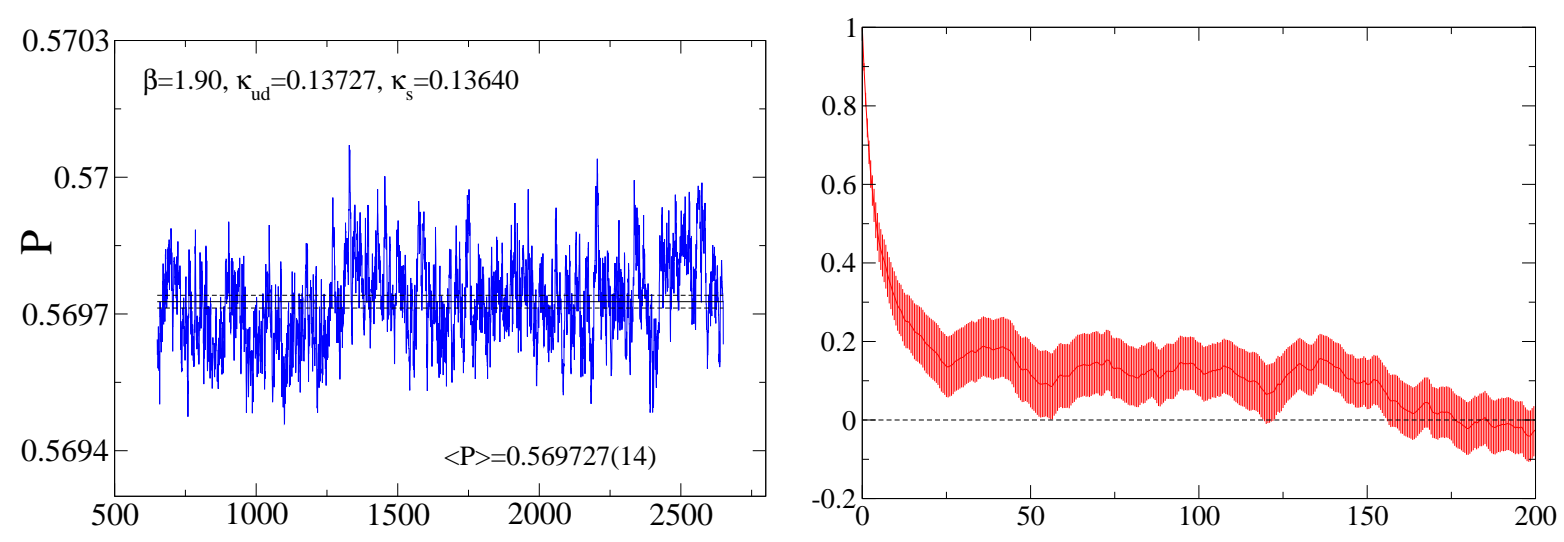

Figure 1: Plaquette history (left) and normalized autocorrelation function (right) for $\left(\kappa_{\mathrm{ud}}, \kappa_{\mathrm{s}}\right)=$ $(0.13727,0.13640)$. 

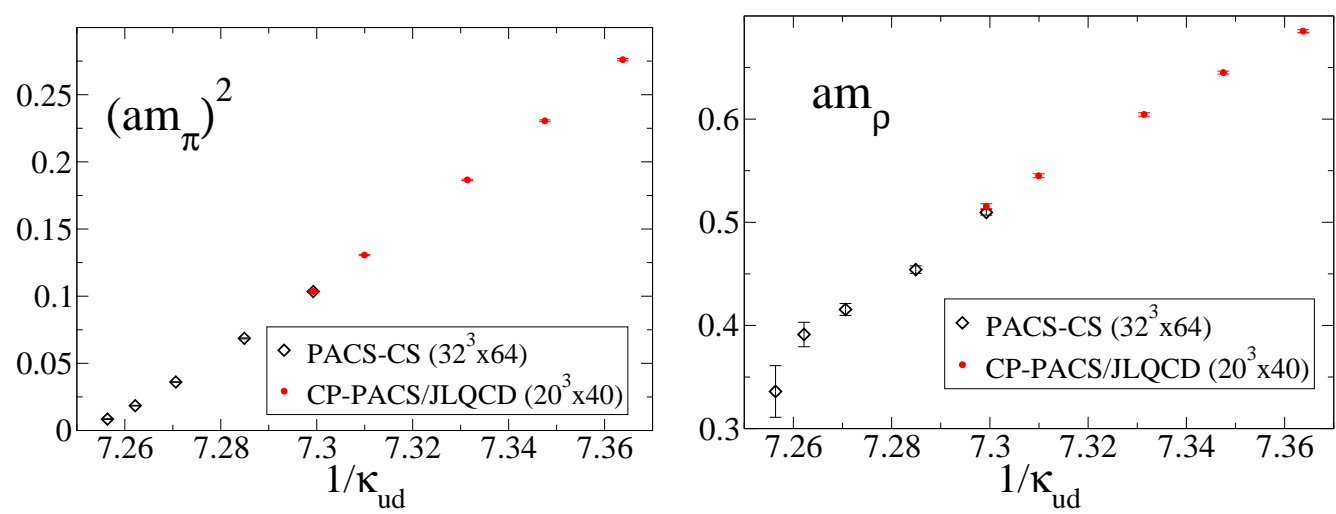

Figure 2: $\left(a m_{\pi}\right)^{2}$ (left) and $a m_{\rho}$ (right) as a function of $1 / \kappa_{\text {ud }}$. Black and red symbols denote the PACS-CS and the CP-PACS/JLQCD results, respectively.

\begin{tabular}{ccccc}
\hline & lattice size & $a m_{\pi}$ & $a m_{\rho}$ & $a m_{\mathrm{N}}$ \\
\hline PACS-CS & $32^{3} \times 64$ & $0.3220(6)$ & $0.506(2)$ & $0.726(3)$ \\
CP-PACS/JLQCD & $20^{3} \times 40$ & $0.3218(8)$ & $0.516(3)$ & $0.733(4)$ \\
\hline
\end{tabular}

Table 2: PACS-CS and CP-PACS/JLQCD results for $\pi, \rho$ and nucleon masses at $\left(\kappa_{\mathrm{ud}}, \kappa_{\mathrm{s}}\right)=$ $(0.13700,0.13640)$.

$\tau_{\text {int }}[P]=20.9(10.2)$ following the definition in Ref. [9]. For other hopping parameters we have found similar behaviors for the normalized autocorrelation function. Although we hardly observe the quark mass dependence for $\tau_{\text {int }}[P]$ listed in Table 1 , the statistics may not be sufficiently large to derive a definite conclusion.

\section{Hadron spectrum}

We measure hadron correlators at every 10 trajectories at the unitary points where the valence quark masses are equal to the sea quark masses. Light hadron masses are extracted from single exponential $\chi^{2}$ fits to the correlators with an exponentially smeared source and a local sink. Statistical errors are estimated by the jackknife method, whose bin size is chosen to be 50 molecular dynamics time based on the bin size dependence of the errors.

We first compare the PACS-CS results on $32^{3} \times 64$ with the previous CP-PACS/JLQCD results on $20^{3} \times 40 \llbracket 1,2 \rrbracket$ at $\beta=1.90$. Figure 4 shows the up-down quark mass dependence of $\left(a m_{\pi}\right)^{2}$ and $a m_{\rho}$ with $\kappa_{\mathrm{s}}$ fixed at 0.13640 . As for the pion mass we observe that the PACS-CS and the CPPACS/JLQCD results are smoothly connected as a function of $1 / \kappa_{\text {ud }}$. Table 2 shows that the results for the pion mass at $\kappa_{\mathrm{ud}}=0.13700$ are consistent within the error bars. On the other hand, we find $1-2 \%$ deviation for the $\rho$ meson and nucleon masses, which could be finite size effects.

Figure 3 shows the hadron effective masses at $\kappa_{\mathrm{ud}}=0.13727$ and 0.13781 . We observe clear plateau for the mesons except for the $\rho$ meson and also good signal for the baryons thanks to a large volume. Especially, the $\Omega$ baryon has a stable signal and a weak up-down quark mass dependence for our simulation parameters. Taking advantage of this virtue we employ the $\Omega$ baryon as input to determine the lattice cutoff. Combined with the additional inputs of $m_{\pi}$ and $m_{K}$ to determine the 

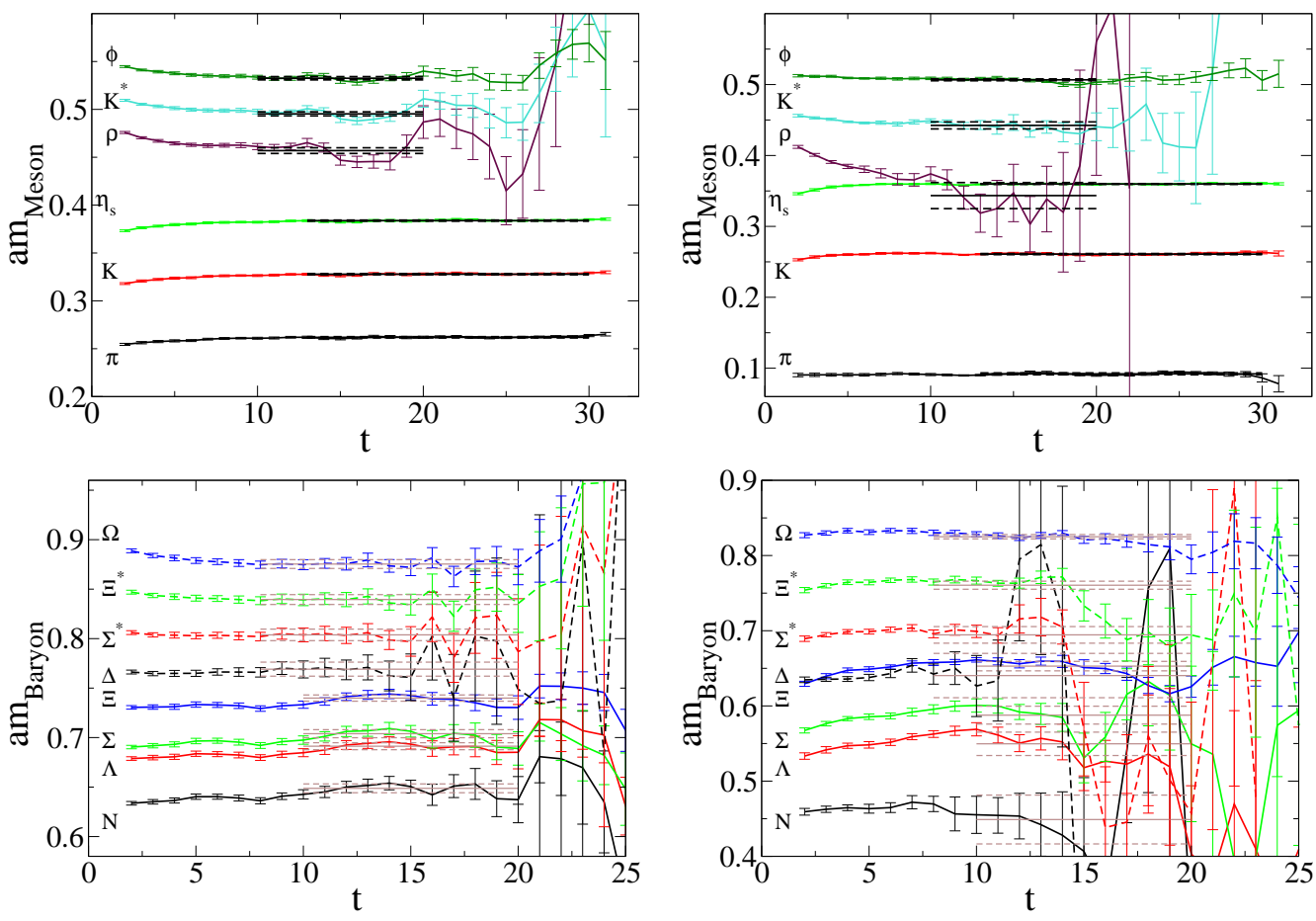

Figure 3: Effective masses for the mesons (top) and the baryons (bottom) at $\kappa_{\mathrm{ud}}=0.13727$ (left) and 0.13781 (right).

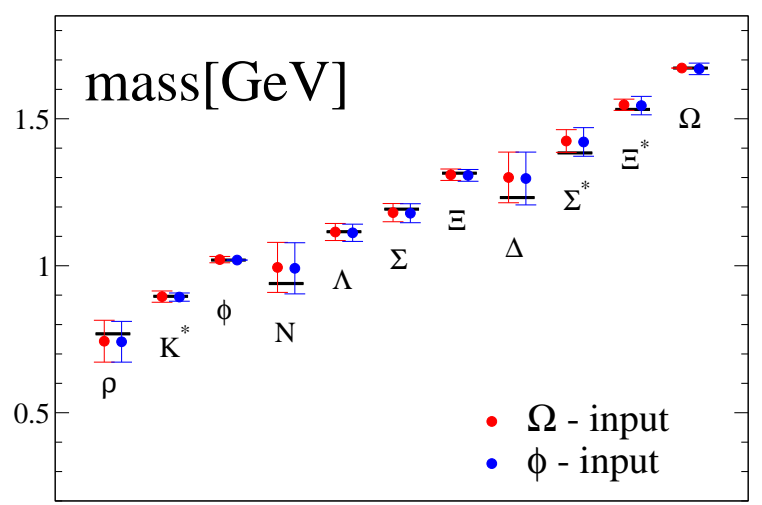

Figure 4: Light hadron spectrum extrapolated at the physical point with $\Omega$-input (red) and $\phi$-input (blue).

physical up-down and strange quark masses, we obtain $a^{-1}=2.256(80) \mathrm{GeV}$. With the use of this cutoff we find that the lightest pseudoscalar meson mass we have reached is about $210 \mathrm{MeV}$. The light hadron spectrum extrapolated to the physical point is shown in Fig. 4, and compared with the experimental values. They show a good agreement within the error bars, though our results contain possible $O\left(\left(a \Lambda_{\mathrm{QCD}}\right)^{2}\right)$ cutoff errors. In Fig. 4 we also plot the results for the $\phi$-input case, which are consistent with the $\Omega$-input case. A more detailed description about the determination of $a^{-1}$ and the chiral extrapolation of the hadron masses is given in Ref. [14].

We calculate the bare quark masses using the axial vector Ward-Takahashi identity (AWI) defined by $a m^{\mathrm{AWI}}=\lim _{t \rightarrow \infty}\left\langle\nabla_{4} A_{4}^{\mathrm{imp}}(t) P(0)\right\rangle /(2\langle P(t) P(0)\rangle)$, where $A_{4}^{\text {imp }}$ is the nonperturbatively 

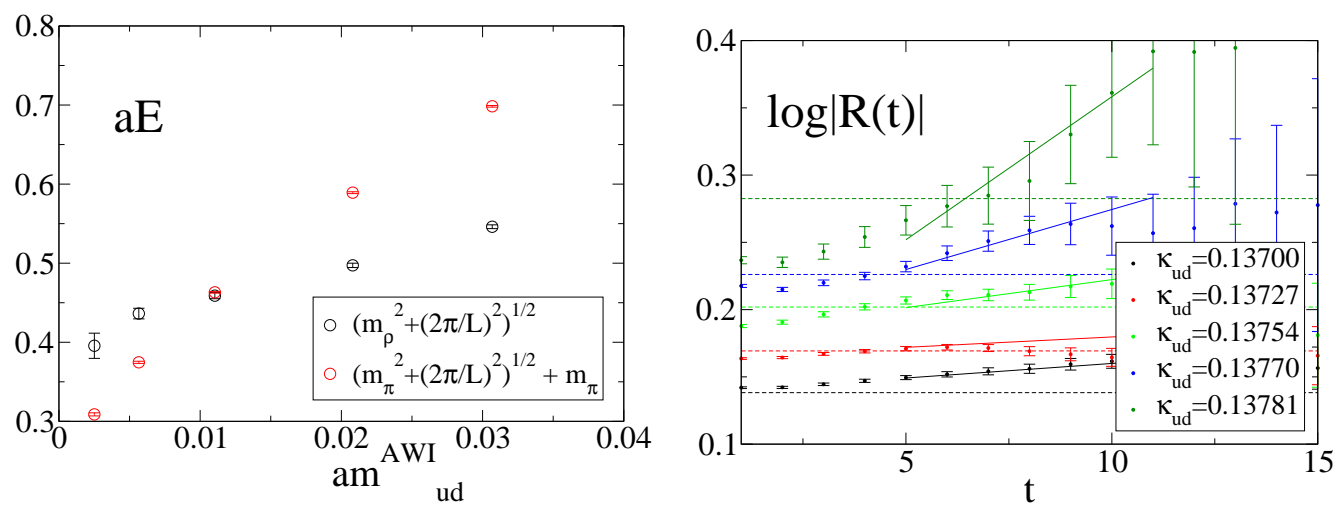

Figure 5: Energy levels of the $\rho$ meson and the two pion states with the momentum $2 \pi / L$ as a function of the up-down quark mass with $\kappa_{s}=0.13640$ (left) and time dependence of the $R$ function (right).

$O(a)$-improved axial vector current [16]. Employing the perturbative renormalization factors $Z_{A, P}$ evaluated up to one-loop level [17] 18], we obtain

$$
m_{\mathrm{ud}}^{\overline{\mathrm{MS}}}(\mu=2 \mathrm{GeV})=2.3(11) \mathrm{MeV}, \quad m_{\mathrm{s}}^{\overline{\mathrm{MS}}}(\mu=2 \mathrm{GeV})=69.1(25) \mathrm{MeV} .
$$

The physical up-down quark mass is about half of our lightest one $m_{\mathrm{ud}}^{\overline{\mathrm{MS}}}(\mu=2 \mathrm{GeV})=5.61(40)$ at $\left(\kappa_{\mathrm{ud}}, \kappa_{\mathrm{s}}\right)=(0.13781,0.13640)$. We also measure the pseudoscalar meson decay constants, for which we find

$$
f_{\pi}=144(6) \mathrm{MeV}, \quad f_{K}=175(6) \mathrm{MeV}, \quad f_{K} / f_{\pi}=1.219(22)
$$

at the physical point with the perturbative $Z_{A}$. They are about $10 \%$ larger than the experimental values, though their ratio, which is free from the ambiguities of the renormalization factors, is consistent with the experimental value. For both the quark masses and the pseudoscalar meson decay constants, our concern is the use of the perturbative renormalization factors which might cause sizable systematic errors. We are now calculating the nonperturbative $Z_{A, P}$ with the Schrödinger functional scheme.

\section{5. $\rho-\pi \pi$ mixing}

Since our simulations are carried out at sufficiently small up-down quark masses, it would be interesting to investigate the $\rho-\pi \pi$ mixing effects [19, 20]. We find that, while the rest mass $m_{\rho}$ is always smaller than the two-pion energy $2 \sqrt{m_{\pi}^{2}+(2 \pi / L)^{2}}$ for all the hopping parameters, the energy of a moving $\rho$ with a unit of momentum, i.e., $\sqrt{m_{\rho}^{2}+(2 \pi / L)^{2}}$, becomes larger than $\sqrt{m_{\pi}^{2}+(2 \pi / L)^{2}}+m_{\pi}$ toward the lighter up-down quark masses. This situation is illustrated in Fig. 5

Let us consider two types of the $\rho$ meson propagator with the momentum $2 \pi / L: \rho_{\|}(2 \pi / L)$ with polarization parallel to the spatial momentum and $\rho_{\perp}(2 \pi / L)$ with polarization perpendicular to the spatial momentum. Phenomenologically the $\rho-\pi \pi$ coupling is described by $g_{\rho \pi \pi} \varepsilon_{a b c} \rho_{\mu}^{a} \pi^{a} \partial_{\mu} \pi^{c}$, which favors $\rho_{\|}(2 \pi / L) \rightarrow \pi(2 \pi / L) \pi(0)$ to $\rho_{\perp}(2 \pi / L) \rightarrow \pi(2 \pi / L) \pi(0)$. We expect that the $\rho_{\|}(2 \pi / L)$ 
propagator is more strongly affected by the mixing effects than the $\rho_{\perp}(2 \pi / L)$ correlator. Since the mixing effects push up the upper energy level further and push down the lower energy level, they could be detected by measuring the $R$ function defined by

$$
R(t)=\frac{\left\langle\rho_{\|}(\vec{p}, t) \rho_{\|}^{\dagger}(\vec{p}, 0)\right\rangle}{\left\langle\rho_{\perp}(\vec{p}, t) \rho_{\perp}^{\dagger}(\vec{p}, 0)\right\rangle} \stackrel{\text { large } t}{\longrightarrow} Z \mathrm{e}^{-\left(E_{\boldsymbol{\rho}_{\|}}-E_{\rho_{\perp}}\right) t} .
$$

In Fig. 5 we plot $\log |R(t)|$ as a function of $t$. The data show clear positive slopes which indicate $E_{\rho_{\|}}<E_{\rho_{\perp}}$. We also observe that the magnitude of the energy difference is rather small for $\kappa_{\text {ud }} \leq$ 0.13754 , while it grows rapidly as the up-down quark mass is reduced for $\kappa_{\mathrm{ud}}>0.13754$. This feature may suggest that the $\left\langle\rho_{\|}(\vec{p}, t) \rho_{\|}^{\dagger}(\vec{p}, 0)\right\rangle$ correlator is getting dominated by the $\pi \pi$ state toward the smaller up-down quark masses. In order to obtain a definite conclusion, we need more detailed investigations with increased statistics.

\section{Acknowledgment}

This work is supported in part by Grants-in-Aid for Scientific Research from the Ministry of Education, Culture, Sports, Science and Technology (Nos. 13135204, 15540251, 17340066, 17540259, 18104005, 18540250, 18740139).

\section{References}

[1] CP-PACS and JLQCD Collaborations, T. Ishikawa et al., PoS (LAT2006) 181.

[2] CP-PACS and JLQCD Collaborations, T. Ishikawa et al., hep-lat/0704.193.

[3] JLQCD Collaboration, S. Aoki et al., Phys. Rev. D73 (2006) 034501,

[4] Y. Iwasaki, preprint, UTHEP-118 (Dec. 1983), unpublished.

[5] PACS-CS Collaboration, S. Aoki et al., PoS (LAT2005) 111.

[6] PACS-CS Collaboration, A. Ukawa et al., PoS (LAT2006) 039.

[7] PACS-CS Collaboration, Y. Kuramashi et al. PoS (LAT2006) 029.

[8] PACS-CS Collaboration, Y. Kuramashi et al., these proceedings.

[9] M. Lüscher, JHEP 0305052 (2003); Comput. Phys. Commun. 156209 (2004); ibid. 165199 (2005)

[10] A. Kennedy, Nucl. Phys. B (Proc. Suppl.) 140 (2005) 190.

[11] L. Del Debbio et al., JHEP 0702056 (2007); JHEP 0702 (2007) 082.

[12] J. C. Sexton and D. H. Weingarten, Nucl. Phys. B380 (1992) 665.

[13] PACS-CS Collaboration, K-I. Ishikawa et al. PoS (LAT2006) 027.

[14] PACS-CS Collaboration, D. Kadoh et al., PoS (LAT2007) 109.

[15] CP-PACS and JLQCD Collaborations, S. Aoki et al., Phys. Rev. D73 (2006) 034501.

[16] CP-PACS/JLQCD and ALPHA Collaborations, T. Kaneko et al., JHEP 0704 (2007) 092.

[17] S. Aoki et al., Phys. Rev. D58, (1998) 074505.

[18] Y. Taniguchi and A. Ukawa, Phys. Rev. D58 114503 (1998).

[19] C. Bernard et al., Phys. Rev. D48, (1993) 4419.

[20] UKQCD Collaboration, C. McNeile and C. Michael, Phys. Lett. B556 (2003) 117. 